\title{
Some theorems and examples on local equiconnectedness and its specializations
}

by

D. W. Curtis (Baton Rouge, La.)

1. Introduction. The property of local equiconnectedness was introduced by Fox [4]. Briefly, a space is locally equiconnected if there exists a jointly continuous selection of paths between nearby points, with the constant path being assigned if the points coincide. This obviously implies local contractibility, and in finite-dimensional metric spaces, these properties are equivalent, though not in general. On the other hand, every metrizable absolute neighborhood retract is locally equiconnected; the converse (for metrizable spaces) remains an open question.

Dugundji [3] and Himmelberg [6] have studied local equiconnectedness an various specializations, principally in metrizable spaces. There are certain additional "local convexity" conditions one can require of local equiconnecting functions, which are sufficient (but not necessary) for obtaining absolute neighborhood retracts. Alternatively, by considering continuous systems of singular simplexes of all dimensions, similar to the "convex structures" of Michael-[8], one obtains a characterization of metrizable absolute neighborhood retracts.

In $\S 2$ of this paper we present proofs of some of the basic results on local equiconnectedness and its specializations in a more general setting. Some theorems on limits of inverse sequences of metrizable absolute neighborhood retracts are established in $\S 3$, and in $\S 4$ various examples of strictly locally equiconnected spaces (including polytopes with the metric and $\mathrm{CW}$ topologies) are given. All spaces considered are Hausdorff.

\section{Local equiconnecting functions and local convex structures.}

Definition (Fox [4]). A local equiconnecting function for a space $Y$ is a map $\lambda: U \times I \rightarrow Y$, where $U$ is a neighborhood of the diagonal in $Y \times Y$, such that $\lambda\left(y_{0}, y_{1}, i\right)=y_{i}, i=0,1$, and $\lambda(y, y, t)=y$, for every $y_{0}, y_{1}, y \in T, t \in I$. 
The $\lambda$-extension of a subset $M \subset Y$ is the smallest non-empty subset $\hat{M} \subset Y$ (if it exists) such that $M \times \hat{M} \subset U$ and $\lambda(M \times \hat{M} \times I) \subset \hat{M} . M$ is $\lambda$-convex if $M=\hat{M}$.

Definimions (Dugundji [3]). A local equiconnecting function $\lambda$ is stable if for every neighborhood $N$ of any point $p$ in $Y$ there exists a neighborhood $M$ such that $\hat{M} \subset N$.

(Himmelberg [6]) A local equiconnecting function $\lambda$ is strict if for every neighborhood $N$ of any point $p$ in $Y$ there exists a convex neighborhood $M$ such that $M \subset N$.

For $U$ an open cover of $Y$, and $n \geqslant 1$, let $Y^{n}\left(น_{)}=\left\{\left(y_{1}, \ldots, y_{n}\right) \in Y^{n}\right.\right.$ : $\left.\left\{y_{1}, \ldots, y_{n}\right\} \subset U \in \mathcal{U}\right\}$, with the relative topology. Let $T^{n-1}$ denote the standard $(n-1)$-simplex in Euclidean $n$-space: $T^{n-1}=\left\{\left(t_{1}, \ldots, t_{n}\right) \in R^{n}\right.$ : $\left.t_{i} \geqslant 0, \sum t_{i}=1\right\}$.

DEFINITION (ef. Himmelberg [6]). A local convex structure for a space $Y$ consists of an open cover $U$ and a sequence of maps $\lambda^{n}: Y^{n}(\mathcal{U}) \times$ $\times T^{n-1} \rightarrow Y, n \geqslant 1$, such that: $t_{m}=0$,

(i) $\lambda^{n}\left(y_{1}, \ldots, y_{n} ; t_{1}, \ldots, t_{n}\right)=\lambda^{n-1}\left(y_{1}, \ldots, \overline{\bar{y}}_{m}, \ldots, y_{n} ; t_{1}, \ldots, \overline{\bar{t}}_{m}, \ldots, t_{n}\right)$ if

(ii) for every neighborhood $N$ of any point $p$ in $Y$ there exists a neighborhood $M$ such that $\lambda^{n}\left(M^{n} \times T^{n-1}\right) \subset N$ for all $n$.

Note that (ii) implies $\lambda^{n}\left(y, \ldots, y ; t_{1}, \ldots, t_{n}\right)=y$, and can be viewed as an equicontinuity requirement for the sequence of maps $\lambda_{s}^{n}$ on the diagonals $\Delta^{n}$.

We shall say that $Y$ is (strictly, stably) LEC if it admits a (strict, stable) local equiconnecting function, and that $Y$ is LCS if it admits a local convex structure.

Proposition 2.1. Strictly LEC implies stably LEC implies LCS implies LEC.

Proof. The first and last assertions are obvious. Suppose $\lambda: U \times I \rightarrow Y$ is a stable local equiconnecting function. Let $\vartheta$ be an open cover of $Y$ by sets $V$ such that $\hat{V}$ exists. For $\left(y_{1}, \ldots, y_{n}\right) \in Y^{n}(\mathcal{V})$, inductively define $\lambda^{n}\left(y_{1}, \ldots, y_{n} ; t_{1}, \ldots, t_{n}\right)=y_{1}$ if $t_{1}=1$, and $\lambda\left(y_{1}, \lambda^{n-1}\left(y_{2}, \ldots, y_{n} ; t_{2} /\left(1-t_{1}\right), \ldots\right.\right.$ $\left.\left.\ldots, t_{n} /\left(1-t_{1}\right)\right), 1-t_{1}\right)$ otherwise. Then $\lambda^{n}\left(V^{n} \times T^{n-1}\right) \subset \hat{V}$ for $n \geqslant 1, V \in V$, and $v,\left(\lambda^{n}\right)$ is a local convex structure on $Y$.

It is known (see below) that LCS does not imply stably LEC. Whether in general LEO implies LCS, or stably LEC implies strictly LEC, are open questions.

While every neighborhood retract of a LEC (LCS) space is LEC (LCS), a stronger condition is required, and is useful, for strictly LEC spaces. A neighborhood retraction $r: N \rightarrow A$ onto a subset $A$ of a strictly LEC space $Y$ is strict if each point of $A$ has arbitrarily small convex neighborhoods (in $Y$ ) which are mapped into themselves by $r$. (A similar condition could be given for stably LEC spaces.)

Propositron 2.2. Every strict neighborhood retract of a strictly LEC space is strictly LEC.

Following the notation of Hanner [5], we shall say that a space $Y$ (not necessarily metrizable) is a neighborhood extension space for the class of metrizable spaces, or NES (M), if every map $f: A \rightarrow Y$ from a closed subset $A$ of a metric space $Z$ into $Y$ can be extended to a neighborhood of $A$. Then $Y$ is an ANR(M) iff it is a metrizable NES( $\mathfrak{M})$.

Proposimion 2.3 (Himmelberg [6]). Every ANR(M) is LCS.

Proof. By a well-known theorem of Kuratowski and Wojdyslawski $[1$, p. 79], the metric space $Y$ can be imbedded as a closed subset of a convex set $Z$ in the Banach space of bounded real-valued maps on $Y$. Since $Z$ is strictly LEC, it is LCS, and since $Y$ is a neighborhood retract of $Z$, it is LCS.

The following theorem was proved also by Himmelberg, in a different manner, for metrizable spaces.

THEOREM 2.4. Every LCS space is NES(M).

Proof. An easy generalization of Dugundji's extension of the Tietze theorem. Let $U,\left(\lambda^{n}\right)$ be a local convex structure on $Y$, and $f: A \rightarrow Y$ a map of a closed subset $A$ of a metric space $Z$ into $Y$. There exists an open cover $\left\{G_{\mu}\right\}$ of $Z \backslash A$, canonical with respect to $Z[1$, pp. 69, 70]. For each set $G_{\mu}$ select a point $a_{\mu} \in A$ such that, for some $g_{\mu} \in G_{\mu}, d\left(g_{\mu}, a_{\mu}\right)$ $<2 d\left(g_{\mu}, A\right)$. For each $a \in A$ let $\nabla(a), N_{s}(a)=\{z \in Z: d(a, z)<s\}$ be neighborhoods of $a$ in $Z$ such that $G_{\mu} \cap \bar{V}(a) \neq \varnothing$ implies $G_{\mu} \subset N_{s}(a)$, and $f\left(N_{3 s}(a) \cap A\right) \subset \bar{U}$ for some $U \in \mathcal{U}$. Set $V=\bigcup\{V(a): a \in A\}$. The nerve $\Re$ of the covering $\left\{G_{\mu} \cap V\right\}$ of $\bigvee A$ is a CW polytope, and there exists a map $\tau: V \backslash A \rightarrow \Re$ such that $\tau^{-1}\left(\right.$ st $\left.p_{\mu}\right) \subset G_{\mu} \cap V$, where $p_{\mu}$ is the vertex of $\mathfrak{N}$ corresponding to $G_{\mu} \cap \nabla$. Totally-order the collection $\left\{p_{\mu}\right\}$ of vertices, and define the map $\eta: \mathfrak{N} \rightarrow Y$ by $\eta\left(\sum_{i}^{k} t_{i} p_{i}\right)=\lambda^{k}\left(f\left(a_{1}\right), \ldots, f\left(a_{k}\right)\right.$; $\left.t_{1}, \ldots, t_{k}\right)$, where $p_{i}<p_{j}$ if $i<j$. It can be verified that $\left\{f\left(a_{1}\right), \ldots, f\left(a_{k}\right)\right\} \subset U$ for some $U \in \mathcal{U}$, so $\eta$ is well-defined. Then $g: \nabla \rightarrow Y$, defined by $g \mid A=f$, $g / V \backslash A=\eta \circ \tau$, is a neighborhood extension of $f$. We need only verify the continuity of $g$ on $A$. Let $a \in A$ and a neighborhood $N$ of $f(a)$ in $Y$ be given. There exists a neighborhood $M$ of $f(a)$ such that $\lambda^{n}\left(M^{n} \times T^{n-1}\right) \subset N$ for all $n$. Choose $s, t>0$ such that $G_{\mu} \cap N_{t}(a) \neq \varnothing$ implies $G_{\mu} \subset N_{s}(a)$, and $f\left(N_{s s}(a)\right) \subset M$. Then $g\left(N_{t}(a) \cap \nabla\right) \subset N$, and $g$ is continuous at $a$.

$\mathrm{A}$ space $Y$ is locally contractible if for every neighborhood $N$ of any point $p$ there exists a neighborhood $M$ which is contractible in $N . Y$ is strictly locally contractible if each point has arbitrarily small neighborhoods contractible in themselves. 
Proposition 2.5. Every (stably) LEC space is (strictly) locally contractible.

Proof. Local contractibility is obvious. Strict local contractibility follows from the fact that the $\lambda$-extension $\hat{M}$ of a subset $M$ is contractible in itself.

Since finite-dimensional locally contractible metric implies $\operatorname{ANR}(\mathfrak{M})$, it follows by (2.3) that such spaces are LCS. Borsuk's example of a locally contractible (but not strictly locally contractible) metric non-ANR has been shown by Dugundji [3] to be non-LEC. Since there exist finitedimensional compact $A R(\mathfrak{M})$ spaces which are not strictly locally contractible [1, pp. 152-155], it follows also by (2.3) that LCS does not imply strict local contractibility.

A (strict, stable) local equiconnecting function $\lambda$ defined on all of $\bar{Y} \times \bar{Y} \times I$ is a (strict, stable) equiconnecting function, and we say that $Y$ is (strictly, stably) EC. Likewise, a local convex structure $U,\left(\lambda^{n}\right)$ on $Y$ is a convex structure if $\bar{Y} \epsilon$ U, and we say that $Y$ is a CS space. Olearly, every (strictly, stably) EC space is contractible and (strictly, stably) LEC, and every OS space is contractible LOS. For a large class of spaces the converses are true.

LeMMa 2.6 (Hanner [5], (12.3)). A map $f: A \rightarrow Y$ from a closed subset $A$ of a normal space $X$ into a contractible space $\bar{Y}$ can be extended to all of $X$ if it can be extended to a neighborhood of $A$.

A space $Y$ is $\omega$-paracompact if $Y^{n}$ is paracompact for all $n \geqslant 1$. The class of $\omega$-paracompact spaces includes all metric spaces, all compact spaces, and all locally countable $\mathrm{CW}$ polytopes. The following theorem was proved by Dugundji [3] for metrizable spaces.

THEOREM 2.7. Every w-paracompact contractible (strictly, stably) LEO space is (strictly, stably) $\mathrm{EC}$, and every w-paracompact contractible LOS espace is OS.

Proof. Only LCS spaces will be considered; the other cases are much easier. Let $\mathcal{U},\left(\lambda^{n}\right)$ be a local convex structure for $Y$, and let $V$ be an open star-refinement of $น$. Then $\overline{Y^{n}(\vartheta)} \subset Y^{n}($ น) for all $n$. We shall inductively construct maps $\Lambda^{n}: Y^{n} \times T^{n-1} \rightarrow Y$, maps $r^{n}: T^{n-1} \rightarrow T^{n-1}$, and open sets $O^{n} \subset Y^{n}, n \geqslant 1$, such that:
(i) $\overline{Y^{n}(\mathcal{V})} \subset O^{n} \subset Y^{n}($ น),
(ii) $r^{n}\left(\mathrm{Bd} T^{n-1}\right) \subset \mathrm{Bd} T^{n-1}$, and $r^{n} / \mathrm{Bd} T^{n-1}$ is the map induced by $r^{n-1}$,
(iii) $\Lambda^{n}\left(y_{1}, \ldots, y_{n} ; t_{1}, \ldots, t_{n}\right)=\lambda^{n}\left(y_{1}, \ldots, y_{n} ; r^{n}\left(t_{1}, \ldots, t_{n}\right)\right)$ if $\left(y_{1}, \ldots, y_{n}\right) \in O^{n}$, if $t_{i}=0$.
Then by (i), (iii), and (iv), $\left(\Lambda^{n}\right)$ will be a convex structure for $Y$. We begin by defining $\Lambda^{1}(y ; 1)=y, r^{1}(1)=1$, and $O^{1}=Y$. Suppose $\Lambda^{k}, r^{k}$, and $O^{k}$ have been defined for $k \leqslant n$. For convenience in defining $A^{n+1}$ and $r^{n+1}$, we consider an arbitrary homeomorphism $h: T^{n} \rightarrow T^{n} \times\{0\} \cup \mathrm{Bd} T^{n} \times I$ $C T^{n} \times I$, such that $h(t)=(t, 1)$ if $t \in \mathrm{Bd} T^{m}$. Let $\pi_{i}: Y^{n+1} \rightarrow Y^{n}$ be the projection parallel to the $i$ th coordinate. Since $Y^{n+1}(U) \cap \bigcap_{1}^{n+1} \pi_{i}^{-1}\left(O^{n}\right)$ is an open neighborhood of $\overline{\bar{Y}^{n+1}(\mathcal{V})}$, we may choose $O^{n+1}$ such that $\overline{\bar{Y}^{n+1}(\mathcal{Y})}$ $\subset O^{n+1}$ and $\overline{0^{n+1}} \subset Y^{n+1}(น) \cap \bigcap_{1}^{n+1} \pi_{i}^{-1}\left(O^{n}\right)$. Let $p: h\left(T^{n}\right) \rightarrow T^{n}$ be the projection map. Define $r^{n+1} / h^{-1}\left(T^{n} \times\{0\}\right)=p \circ h, r^{n+1} / h^{-1}\left(\operatorname{Bd} T^{n} \times\left[\frac{1}{2}, 1\right]\right)$ $=r^{n} \circ p \circ h\left(r^{n}: \operatorname{Bd} T^{n} \rightarrow \mathrm{Bd} T^{n}\right.$ is the map induced by $\left.r^{n}: T^{n-1} \rightarrow T^{n-1}\right)$, and extend $r^{n+1}$ "linearly" to $h^{-1}\left(\mathrm{Bd} T^{n} \times\left[0, \frac{1}{2}\right]\right)$. We first define $\Lambda^{n+1}$ on a neighborhood of $\overline{0^{n+1}} \times T^{n} \cup Y^{n+1} \times \mathrm{Bd} T^{n}$ as follows: $\Lambda^{n+1}(y ; t)$ $=\lambda^{n+1}\left(y ; r^{n+1}(t)\right)$ if $y \in \bar{Y}^{n+1}(น) \frown \bigcap_{1}^{n+1} \pi_{i}^{-1}\left(O^{n}\right), \Lambda^{n+1}(y ; t)=\Lambda^{n+1}(y ; p \circ h(t))$ if $t \in h^{-1}\left(\mathrm{Bd} T^{n} \times\left[\frac{1}{2}, 1\right]\right)$, and $A^{n+1} / \Gamma^{1}+1 \times \mathrm{Bd} T^{n}$ is defined by condition (iv). By (2.6), $\Lambda^{n+1}$ can be extended to $Y^{n+1} \times T^{n}$, and conditions (iii) and (iv) are satisfied.

The lemma and theorem to follow were proved by Hanner for $\operatorname{ANR}(\mathfrak{M})$ spaces, and by Himmelberg for metrizable LEC spaces.

LEMVIA 2.8. Let $Y=Y_{1} \cup Y_{2}$ be normal, with each $Y_{i}$ open and LCS (LEC). Then $Y$ is LCS (LEC).

Proof. Again, we consider only LCS spaces. Let $\mathcal{U}_{i},\left(\lambda_{i}^{n}\right), i=1,2$, be local convex structures for $Y_{1}, Y_{2}$. There exist closure-disjoint open sets $Z_{1}, Z_{2}$ in $Y$ with $I \backslash Y_{2} \subset Z_{1}$ and $Y \backslash Y_{1} \subset Z_{2}$. For each $y \in \mathbb{Y} \backslash\left(Z_{1} \cup Z_{2}\right)$, let $U_{y}$ be an open neighborhood of $y$ such that for some $\nabla_{1} \in U_{1}$ and $\bar{U}_{2} \in U_{2}, \bar{U}_{y} \subset U_{1} \cap \bar{U}_{2}$ and $\lambda_{i}^{n}\left(U_{y}^{n} \times T^{n-1}\right) \subset U_{1}$ for all $n \geqslant 1, i=1,2$. Take $\mathcal{U}_{=}=\left\{U_{1} \cap Z_{1}: U_{1} \in \mathcal{U}_{1}\right\} \cup\left\{U_{2} \cap Z_{2}: U_{2} \in \mathcal{U}_{2}\right\} \cup\left\{U_{y}: y \in \bar{Y} \backslash\left(Z_{1} \cup Z_{2}\right)\right\}$, and let $r: Y \rightarrow I$ be a map such that $r\left(\bar{Z}_{1}\right)=0$ and $r\left(\bar{Z}_{2}\right)=1$. For each $n \geqslant 1$, define $\lambda^{n}: Y^{n}\left(\right.$ น $\times T^{n-1} \rightarrow Y$ as follows:

$$
\lambda^{n}\left(\left(y_{i}\right) ;\left(t_{i}\right)\right)=\left\{\begin{array}{lr}
\lambda_{1}^{n}\left(\left(y_{i}\right) ;\left(t_{i}\right)\right), \quad \sum t_{i} r\left(y_{i}\right)=0, \\
\lambda_{1}^{2}\left(\lambda_{1}^{n}\left(\left(y_{i}\right) ;\left(t_{i}\right)\right),\right. & \left.\lambda_{2}^{n}\left(\left(y_{i}\right) ; \quad\left(t_{i}\right)\right) ; 1-\sum t_{i} r\left(y_{i}\right), \sum t_{i} r\left(y_{i}\right)\right), \\
\lambda_{2}^{n}\left(\left(y_{i}\right) ;\left(t_{i}\right)\right), & \sum t_{i} r\left(y_{i}\right)=1 .
\end{array}\right.
$$

It remains an open question whether the above result can be extended to stably or strictly LEC spaces.

THEOREM 2.9. Every completely normal paracompact space which is locally LCS (LEC) is LCS (LEC). 
Proof. An immediate consequence of Michael's theorem on 9 -hereditary local properties [7], the preceding lemma, and the facts that open subsets, and discrete sums, of LCS (LEC) spaces are LCS (LEC).

3. Inverse sequences of $\operatorname{ANR}(\mathfrak{M})$ spaces. We shall say that a series $\sum u_{i}$ of open covers of a space $Y$ converges if every sequence $\left(y_{i}\right)$ in $Y$, for which $\left\{y_{i}, y_{i+1}\right\} \subset U_{i} \in \mathcal{U}_{i}$ for all $i$, converges. If $U$ is an open cover such that, for every sequence $\left(y_{i}\right)$ satisfying the above condition, $\left\{y_{1}, \lim y_{i}\right\}$ $C U \epsilon \mathcal{U}$, we shall say that $\sum \mathcal{U}_{i}$ is subordinate to $น$.

LeMMa 3.1. Let $Y$ be a complete metric space, and $\mathcal{U}$ an open cover. Then there exists a convergent series $\sum \mathcal{U}_{i}$ of open covers subordinate to $\mathcal{W}_{\text {. }}$ Conversely, if $\bar{Y}$ is paracompact and there exists a convergent series of open: covers, then $Y$ is completely metrizable.

Proof. Let $\varrho(x, y)$ be a complete metric on $Y$. Let $\vartheta=\left\{V_{a}\right\}$ be an open locally finite refinement of $\mathcal{U}$, and shrink $\left\{V_{a}\right\}$ to an open cover $\left\{W_{a}\right\}$ such that $\bar{W}_{\alpha} \subset V_{\alpha}$, for all $\alpha$. Let $\left\{f_{\alpha}: I \rightarrow I\right\}$ be a collection of maps such that $f_{a}\left(W_{a}\right)=1, f_{a}\left(T \backslash V_{a}\right)=0$. Define a continuous pseudo-metric $\varrho_{0}$ on $Y$ by $\varrho_{0}(x, y)=\sup \left|f_{\alpha}(x)-f_{a}(y)\right|$. Then $d(x, y)=\varrho(x, y)+\varrho_{0}(x, y)$ is a complete metric on $Y$, topologically equivalent to $\varrho(x, y)$, and the covering by open unit balls of $(Y, d)$ refines $\mathcal{U}$ (cf. Michael [7]). Take $\mathcal{U}_{i}$ to be the covering by open balls of $(Y, d)$ of radius $2^{-i}$.

Conversely, suppose $\sum u_{i}$ is a convergent series of open covers. We may assume each $\mathcal{U}_{i}$ is locally finite. Then $\left[\mathcal{L}_{2 i-1}^{i}, \mathcal{U}_{2 i}\right]=\left\{U_{2 i-1} \cap U_{2 i}\right.$ : $\left.U_{k} \in \mathcal{U}_{k}\right\}$ is also locally finite, for all $i$. We claim that $\bigcup_{1}^{\infty}\left[น_{2 i-1}, น_{2 i}\right]$ is a base for $Y$. If not, there exists $x \in U, U$ open, and a sequence $\left(U_{i}\right)$, $U_{i} \in \mathcal{U}_{i}$, such that $x \in U_{i}$ and $U_{2 i-1} \cap U_{2 i} \backslash U \neq \varnothing$ for each $i$. Choose $x_{2 i} \in U_{2 i-1} \cap U_{2 i} \backslash U$. Then the sequence $\left(x_{i}\right)$, where $x_{2 i-1}=x$, must converge, which is impossible. Thus $Y$ has a $\sigma$-locally finite base and is metrizable. For each $n$, let $\varrho_{n}(x, y)$ be a compatible metric for $Y$ such that $\left\{N \varrho_{n}\left(y ; 2^{-n}\right): y \in Y\right\}$ refines $\mathcal{U}_{n}$. Then $\varrho(x, y)=\sum_{1}^{\infty} \min \left\{\varrho_{n}(x, y), 2^{-n}\right\}$ is a compatible metric for $Y$ such that for each $n$, the covering $V_{n}$ by open balls of radius $2^{-n}$ refines $\mathcal{U}_{n}$. Thus $\sum \mho_{n}$ is a convergent series, and every Cauchy sequence in $(Y, \varrho)$ has a convergent subsequence.

Let $U$ be an open cover of a space $Y$, and consider maps $f, g: X \rightarrow Y$. We say that $f$ and $g$ are $\mathcal{U}$-homotopic if there exists a homotopy $H$ : $X \times I \rightarrow Y$ such that $H(p, 0)=f(p), H(p, 1)=g(p)$, and $H(p \times I) \subset U_{p} \in \mathcal{U}$, for every $p \in X$. Let $f^{-1}(\mathcal{U})=\left\{f^{-1}(U): U \in \mathcal{U}\right\}$.

Defintmion. A map $f: X \rightarrow Y$ is a fine homotopy equivalence if for every open cover $U$ of $Y$, there exists a map $g: X \rightarrow X$ such that $f \circ g$ is $\mathcal{U}$-homotopic to $\mathrm{id}_{Y}$, and $g \circ f$ is $f^{-1}\left(\mathcal{U}_{)}\right.$-homotopic to $\mathrm{id} X$.
THEOREM 3.2. Let $Y=\lim \left(Y_{i}, f_{i+1}\right)$ be the limit of an inverse sequence

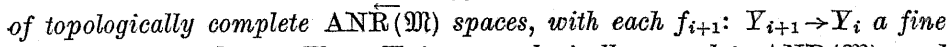
homotopy equivalence. Then $Y$ is a topologically complete $\operatorname{ANR}(\mathfrak{M})$, and the projection maps are fine homotopy equivalences.

Proof. Let $น_{i},\left(\lambda_{i}^{n}\right)$ be local convex structures for the spaces $Y_{i}$, and for $A \subset Y_{i}$, define $\hat{\lambda}_{i}(A)=\bigcup\left\{\lambda_{i}^{n}\left(A^{n}\left(\mathcal{U}_{i}\right) \times T^{n-1}\right): n \geqslant 1\right\}$. We inductively construct sequences of maps $g_{i}: Y_{i} \rightarrow Y_{i+1}$, homotopies $H_{i}$ : $Y_{i} \times I \rightarrow Y_{i}(i>1)$, and open covers $v_{i}, w_{i},\left(w_{i}^{n}: n \geqslant i\right)$ of $Y_{i}$, such that:

(i) $\bar{\vartheta}_{i}=\left\{\bar{V}: V \in \mho_{i}\right\}$ is a locally finite refinement of $\mathcal{U}_{i}$, and $\vartheta_{i}$ is a refinement of $\left(f_{i-k+1} \circ \ldots \circ f_{i}\right)^{-1}\left(w_{i-k}^{i}\right)$ for each $1 \leqslant k<i$,

(ii) $w_{i}$ is a locally finite star-refinement of a star-refinement of $v_{i}$,

(iii) $\sum w_{i}^{n}$ is a convergent series of open covers subordinate to a starrefinement of $w_{i}$, and mesh $w_{i}^{n} \leqslant 2^{-n}$,

(iv) $f_{i+1} \circ g_{i}$ is $w_{i}^{i}$-homotopic to id $Y_{i}$, and $H_{i+1}$ is a $f_{i+1}^{-1}\left(w_{i}^{i}\right)$-homotops, with $H_{i+1}(y, 0)=g_{i} \circ f_{i+1}(y)$ and $H_{i+1}(y, 1)=y$,

(v) for every $V \epsilon V_{i+1}$ and $t \epsilon I, \hat{\lambda}_{i}\left(\hat{\lambda}_{i}\left(f_{i+1} \circ H_{i+1}\left(\hat{\lambda}_{i+1}(\bar{V}), t\right)\right)\right) \subset W \epsilon W_{i}^{i}$.

With $p_{j}: Y \rightarrow Y_{j}$ the projection map, define $q_{i}: Y_{i} \rightarrow Y$ by $p_{j} \circ q_{i}$ $=\lim _{n \rightarrow \infty} f_{i+1} \circ \ldots \circ f_{n+1} \circ g_{n} \circ \ldots \circ g_{i}$. It can be verified that $q_{i}$ is a well-defined map, and $q_{i+1} \circ g_{i}=q_{i}$. Define a homotopy $H: Y \times[1, \infty] \rightarrow Y$ as follows:

$$
\begin{aligned}
& H(y, i+s)= \begin{cases}q_{i} p_{i}(y), & 0 \leqslant s \leqslant \frac{1}{2}, \\
q_{i+1} H_{i+1}\left(p_{i+1}(y), 2 s-1\right), & \frac{1}{2} \leqslant s \leqslant 1,\end{cases} \\
& H(y, \infty)=y .
\end{aligned}
$$

With $\vartheta=p_{1}^{-1}\left(\vartheta_{1}\right)$, let $\widetilde{F}=\{F \subset Y: F$ is finite non-empty and $F \subset \bar{V} \in \overline{\mathcal{V}}\}$. A level indicator $\omega$ for $F^{F} \in \tilde{\mho}$ is a function $\omega: 2^{F} \backslash\{\emptyset\} \rightarrow[1, \infty]$ such that:

(i) for $A \subset B \subset F, \omega(A) \geqslant \omega(B)$,

(ii) for $A \subset F$ non-trivial, $\omega(A) \leqslant i$ and $p_{i}(A) \subset \bar{V} \in \bar{\vartheta}_{i}$ for some $i \geqslant 1$. Let $\widetilde{F}^{n}=\left\{\left(y_{1}, \ldots, y_{n} ; \omega\right):\left\{y_{1}, \ldots, y_{n}\right\} \in \mathfrak{F}\right.$ and $\omega$ is a level indicator for $\left.\left\{y_{1}, \ldots, y_{n}\right\}\right\}$, with the topology of point-wise convergence (product topology). Define a sequence of maps $\Lambda_{0}^{n}: \mathbb{\mho}^{n} \times T^{n-1} \rightarrow Y, n \geqslant 1$, as follows:

$$
\begin{aligned}
& \Lambda_{0}^{n}\left(y_{1}, \ldots, y_{n} ; t_{1}, \ldots, t_{n} ; \omega\right) \\
& =\left\{\begin{array}{c}
y_{1}\left(=y_{2}=\ldots=y_{n}\right), \omega\left\{y_{1}, \ldots, y_{n}\right\}=\infty, \\
q_{i+1} H_{i+1}\left(\lambda_{i+1}^{n}\left(p_{i+1}\left(y_{1}\right), \ldots, p_{i+1}\left(y_{n}\right) ; t_{1}, \ldots, t_{n}\right), 2 s-1\right), \\
\omega\left\{y_{1}, \ldots, y_{n}\right\}=i+s, \quad \frac{1}{2} \leqslant s \leqslant 1, \\
q_{i} \lambda_{i}^{2}\left(f_{i+1}\left(\lambda_{i+1}^{n}\left(p_{i+1}\left(y_{1}\right), \ldots, p_{i+1}\left(y_{n}\right) ; t_{1}, \ldots, t_{n}\right)\right), \lambda_{i}^{n}\left(p_{i}\left(y_{1}\right), \ldots, p_{i}\left(y_{n}\right) ;\right.\right. \\
\left.\left.t_{1}, \ldots, t_{n}\right) ; 2 s, 1-2 s\right), \omega\left\{y_{1}, \ldots, y_{n}\right\}=i+s, \quad 0 \leqslant s \leqslant \frac{1}{2} .
\end{array}\right.
\end{aligned}
$$


We shall inductively construct a sequence of maps $\Lambda^{n}: \mathfrak{F}^{n} \times \dot{T}^{n-1} \rightarrow Y$ such that:

(i) $\Lambda^{n}\left(y_{1}, \ldots, y_{n} ; t_{1}, \ldots, t_{n} ; \omega\right)=\Lambda_{0}^{n}\left(y_{1}, \ldots, y_{n} ; t_{1}, \ldots, t_{n} ; \omega\right)$ if $\omega\left\{y_{1}, \ldots, y_{n}\right\}$ $=\max \left\{\omega\left(y_{i}\right)\right\}$

(ii) $\Lambda^{n+1}\left(y_{1}, \ldots, y_{n+1} ; t_{1}, \ldots, t_{n+1} ; \omega\right)=\Lambda^{n}\left(y_{1}, \ldots, \overline{\bar{y}}_{i}, \ldots, y_{n+1} ; t_{1}, \ldots\right.$, $\left.\overline{\bar{t}}_{i}, \ldots, t_{n+1} ; \omega\right)$ if $t_{i}=0$.

Suppose $\Lambda^{n}$ has been defined; let $\left(y_{1}, \ldots, y_{n+1} ; t_{1}, \ldots, t_{n+1} ; \omega\right)$ in $\mathfrak{F}^{n+1} \times T^{n}$ be given. Let $e_{i} \in T^{n}$ such that $e_{t}(j)=1$ if $i=j, 0$ otherwise. With $k=\left(\max \left\{\omega\left(y_{i}\right)\right\}-\omega\left\{y_{1}, \ldots, y_{n+1}\right\}\right)^{-1}$, let ${ }^{k} T^{n}$ denote the convex hull of $\left\{\tilde{e}_{1}, \ldots, \tilde{e}_{n+1}\right\} \subset T^{n}$, where $\tilde{e}_{i}(j)=(k+2) /(n+k+2)$ if $i=j, 1 /(n+k+2)$ otherwise. Consider $T^{n}={ }^{k} T^{n} \cup \mathrm{Bd}^{k} T^{n} \times[k, \infty]$, with $\mathrm{Bd}^{k} T^{n} \times\{k\}=\mathrm{Bd}^{k} T^{n}$, and $\mathrm{Bd}^{k} T^{n} \times\{\infty\}=\mathrm{Bd} T^{n}$. For the given point $\left(t_{1}, \ldots, t_{n+1}\right)=\sum t_{j} e_{j}$ in $T^{n}$, there are two possibilities:

(i) if $\sum t_{j} e_{j}=\sum_{\tilde{t}} \tilde{t}_{j} \tilde{e}_{j} \epsilon{ }^{k} T^{n}$, define $\Lambda^{n+1}\left(y_{1}, \ldots, y_{n+1} ; t_{1}, \ldots, t_{n+1} ; \omega\right)$ $=\Lambda_{0}^{n+1}\left(y_{1}, \ldots, y_{n+1} ; \tilde{t}_{1}, \ldots, \tilde{t}_{n+1} ; \omega\right)$,

(ii) if $\sum t_{j} e_{j}=\left(\sum\left\{\tilde{t}_{j} \tilde{\epsilon}_{j}: j \neq i\right\}, q\right) \in \mathrm{Bd}^{k} T^{n} \times[k, \infty]$, define $\Lambda^{n+1}\left(y_{1}, \ldots\right.$, $\left.y_{n+1} ; t_{1}, \ldots, t_{n+1} ; \omega\right)=\Lambda^{n}\left(y_{1}, \ldots, \overline{\bar{y}}_{i}, \ldots, y_{n+1} ; \tilde{t}_{1}, \ldots, \overline{\bar{t}_{i}}, \ldots, \tilde{t}_{n+1} ; \omega_{q}\right)$, where $\omega_{q}(A)=(q-k) \omega(A) /(q+1)+(k+1) \omega\left\{y_{1}, \ldots, y_{n+1}\right\} /(q+1)$.

For non-trivial $F \in \widetilde{F}$, there is a largest integer $i \geqslant 1$ such that $p_{i}(F)$ $\subset \bar{V} \in \bar{v}_{i}$. Let $v=\max \left\{d\left(p_{i}(F), Y_{i} \backslash V\right): \bar{V} \in \mathcal{V}_{i}\right\}$, and $w=\inf \left\{\max \left\{d\left(p_{i}(a)\right.\right.\right.$, $\left.\bar{W}): a \in F\}: W \in W_{i}\right\}$. We define a level indicator map $m: \mathfrak{F} \rightarrow[1, \infty]$ as follows ( $\widetilde{F}$ is given the Hausdorff metric for subsets): $m(F)=\infty$ if $F$ is trivial, $i-w /(v+w)$ otherwise. It is clear that for $F \in \mathfrak{F}, \omega: 2^{F} \backslash\{\emptyset\} \rightarrow[1, \infty]$ defined by $\omega(A)=m(A)$ is a level indicator.

For each $n \geqslant 1$, define $\lambda^{n}: Y^{n}(\mathcal{U}) \times T^{n-1} \rightarrow Y$ by $\lambda^{n}\left(y_{1}, \ldots, y_{n} ; t_{1}, \ldots, t_{n}\right)$ $=\Lambda^{n}\left(y_{1}, \ldots, y_{n} ; t_{1}, \ldots, t_{n} ; \omega\right)$, where $\omega(\boldsymbol{A})=m(\boldsymbol{A})$ for every $A \subset\left\{y_{1}, \ldots, y_{n}\right\}$. We show that $\vartheta,\left(\lambda^{n}\right)$ is a local convex structure. For $S \subset Y$ with $p_{i+1}(S)$ $\subset \bar{V} \in \bar{V}_{i+1}$ and $i \leqslant m \leqslant i+1$ for some $i \geqslant 1$, let $\hat{\Lambda_{0}}(S, m)=\cup\left\{\Lambda_{0}^{n}\left(S^{n}\right.\right.$ $\left.\left.T^{n-1}, \omega\right): n \geqslant 1\right\}$, where $\omega(A)=m$ for each finite $A \subset S$. Then $p_{i}\left(\hat{\Lambda_{0}}(S, m)\right)$ $\subset W \in w_{i}$, and $\hat{\lambda}(S)$ is contained in the union of sets of the form $\hat{\Lambda}_{0}(R, q)$, where $R \subset S, p_{j+1}(R) \subset \bar{V} \in \overline{\mathcal{V}}_{j+1}$, and $i \leqslant j \leqslant q \leqslant j+1$. For each such pair $(R, q)$, we have $p_{i}\left(\hat{\Lambda}_{0}(R, q)\right) \subset W \in W_{i}$. Since $H(R, q) \subset \hat{\Lambda}_{0}(R, q)$, since $\left\{p_{i}(H(y, q)), p_{i}(y)\right\} \subset W \in W_{i}$ for every $y \in Y$, and since $p_{i}(S) \subset W$ $\epsilon \mathcal{W}_{i}$, we have $p_{i}(\hat{\lambda}(S)) \subset V \epsilon \mathcal{V}_{i}$. Thus $Y$ admits a local convex structure and is therefore an $\operatorname{ANR}(\mathfrak{M})$.

Theorem 3.2 is also true for completely metrizable LEC spaces, with the proof a simplification of that presented above. The requirement of fine homotopy equivalences is essential-there are easy examples of non-locally connected tree-like continua with bonding maps deformation
retractions.
LEMMA 3.3. A retraction $f: X \rightarrow Y$ onto a paracompact space $Y$ is a fine homotopy equivalence iff for every open cover $U$ of $Y, f: X \rightarrow X$ is $f^{-1}\left(\right.$ () -homotopic to $\mathrm{id}_{X}$.

Proof. Only the necessity of the condition requires proof. Let $\vartheta$ be a barycentric refinement of $น$. There exists a map $g: Y \rightarrow X$, and a $f^{-1}(\mathcal{Y})$ homotopy $G: X \times I \rightarrow X$ with $G(x, 0)=x, G(x, 1)=g f(x)$. Define $H$ : $X \times I \rightarrow X$ by $H(x, t)=G(x, 2 t)$ if $0 \leqslant t \leqslant \frac{1}{2}$, and $H(x, t)=G(f(x), 2-2 t)$ if $\frac{1}{2} \leqslant t \leqslant 1$. Then $H$ is a $f^{-1}(u)$-homotopy between $f: X \rightarrow X$ and $\mathrm{id}_{X}$.

A retraction which is a fine homotopy equivalence will be called a fine deformation retraction.

CoRollary 3.4. The limit of an inverse sequence of ANR(M) spaces, with bonding maps fine deformation retractions, is an $\operatorname{ANR}(\mathfrak{M})$, and the projection maps are fine deformation retractions.

Proof. This is a corollary of the proof of (3.2), and holds also for metrizable LEC spaces. The hypothesis of completeness was used only in obtaining, for each $i \geqslant 1$, the convergent series of open covers $\sum w_{i}^{n}$. But this convergence property is unnecessary when the maps $f_{i+1}$ are retractions. The maps $g_{i}: Y_{i} \rightarrow Y_{i+1}, q_{i}: Y_{i} \rightarrow Y$ may be chosen to be the inclusion maps. The sequence $\left(w_{i}^{n}: n \geqslant i\right)$ may be chosen to be any sequence of open refinements of $w_{i}$ such that mesh $w_{i}^{n} \leqslant 2^{-n}$.

A retraction $f: X \rightarrow Y$ is a straight deformation retraction if there exists a homotopy $H: X \times I \rightarrow X$ between $f$ and $\operatorname{id}_{X}$ such that $f H(x, t)$ $=f(x)$ for all $x, t$. Obviously, $f$ is then a fine deformation retraction. It can be shown that the limit of an inverse sequence of metrizable stably LEC spaces, with bonding maps straight deformation retractions, is stably LEC. We give an example of a fine deformation retraction $r$ : $|P| \rightarrow|Q|$ of a contractible finite complex $|P|$ onto a subcomplex $|Q|$ which is not a straight deformation retraction. Let $P$ be the complex in $R^{3}$ with 2 -simplices $P_{1}, P_{2}, P_{3}, P_{4}$ spanned by the sets $\{(0,0,0),(0,1,0)$, $(0,1,1)\},\{(0,0,0),(0,1,1),(1,1,0\},\{(0,1,0),(0,1,1),(1,1,0)\}$ and $\{(0,1,0),(1,1,0),(0,2,0)\}$, respectively, and let $Q$ be the subcomplex with 1-simplices $Q_{1}, Q_{2}$ spanned by $\{(0,0,0),(0,1,0)\},\{(0,1,0),(0,2,0)\}$, respectively. Topologically, $|P|$ is a 2 -cell and $|Q|$ an arc in its boundary. The retraction $r:|P| \rightarrow|Q|$ is defined by $r(x, y, z)=(0, y, 0)$.

4. Some examples of strictly LEC spaces. While every convex subset of a (locally convex) topological linear space is (strictly) EC, it is not clear whether every topological linear space, or even every metrizable linear space, admits a convex structure.

We show here that all polytopes with the metric topology, and a large class of polytopes with the OW topology (including all 1-dimensional 
and all locally countable polytopes) are strictly LEC. Hanner [5] observed that all metric polytopes are $\operatorname{NES}(\mathfrak{M})$ spaces, and Hanner and Dugundji [2] independently obtained the same result for all CW polytopes.

LEMCMA 4.1. Every full metric polytope is strictly EC.

Proof. We may regard such a polytope $|K|$ as the convex hull of the elements of an orthonormal basis for a suitable Hilbert space.

LEMva 4.2. Every subpolytope $|L|$ of a full metric polytope $|K|$ is a strict neighborhood retract of $|K|$.

Pro of. We use the standard neighborhood retraction. Since the barycentric subdivision $\mathrm{sd} L$ is full in $\mathrm{sd} K$, and since the identity map ( $|\mathrm{sd} K|$, $|\operatorname{sd} L|) \rightarrow(|K|,|L|)$ is a homeomorphism, the map $r: \bigcup\{\operatorname{st} v: v \in \operatorname{sd} L\} \rightarrow|\operatorname{sd} L|$ defined by $r(a x+(1-a) y)=x$, where $0<a \leqslant 1, x \in \sigma \in \mathrm{sd} L$, and $y \in \tau$ $\epsilon \operatorname{sd} K$ such that $|\tau| \cap|L|=\varnothing$, is a neighborhood retraction onto $|L|$. (Here, and in what follows, $\tau$ is the open simplex, $|\tau|$ the closed simplex). We use the metric $d\left(\left(x_{a}\right),\left(y_{\alpha}\right)\right)=\sum\left|x_{a}-y_{a}\right|$ on $|\operatorname{sd} K|$, where the $x_{\alpha}$ are the barycentric coordinates of $x=\left(x_{a}\right)$ in the polytope sd $K$. For $x \epsilon|s \mathrm{~d} L|$ and $t>0$, let $N(x ; t)=\{y €|s d K|: d(x, y)<t\}$. Rather tedious but straight-forward computations show that $N(x ; t)$ is a convex subset of $|K|$ and that $r(N(x ; t)) \subset N(x ; t)$, assuming $t$ is small enough.

THEOREM 4.3. Every metric polytope is strictly LEC.

Proof. Every polytope $|L|$ can be regarded as a subpolytope of a full polytope $|K|$, and the result follows from (4.1), (4.2), and $(2.2)$.

LEMMA 4.4. A countable-dimensional linear space $E$ with the finite topology is a locally convex topological linear space.

Proof. Let $\left\{u_{i}\right\}$ be a basis for $E$, and for each $n$, let $L_{n}=\left\{t u_{n}:-\infty\right.$ $<t<\infty\}$. Let $U$ be an open neighborhood of 0 . Take $V_{1}$ to be an open symmetric convex subset of $L_{1}$ such that $0 \in \bar{V}_{1} \subset U$. Inductively, take $V_{n}$ to be an open symmetric subset of $L_{n}$ such that $\overline{\operatorname{conv}}\left(\bigcup_{1}^{n} V_{i}\right) \subset U$. Then $\operatorname{conv}\left(\bigcup_{1}^{\infty} \nabla_{i}\right) \subset U$ is an open symmetric convex neighborhood of $O$. Since scalar multiplications $x \rightarrow a x$ and translations $x \rightarrow x+x_{0}$ are continuous on finite-dimensional flats, and therefore on $E$, and since the symmetric convex neighborhoods of $O$ form a local base, $E$ is a locally convex topological linear space:

COROLLART 4.5. Every full countable CW polytope is strictly EC.

Proof. We may regard such a polytope $|K|$ as the convex hull of the elements of a basis for a countable-dimensional linear space with the finite topology.
LeMna 4.6. Every subpolytope $|L|$ of a full countable $\mathrm{CW}$ polytope $|K|$ is a strict neighborhood retract of $|K|$.

Proof. Again, we use the standard neighborhood retraction $r: \bigcup$ st $v$ : $v \in \operatorname{sd} L\} \rightarrow|\operatorname{sd} L|$. Let $x \in \sigma_{0} \in \operatorname{sd} L$, and let $U \subset|K|$ be an open neighborhood of $x$. Let $V_{0}$ be a relatively open convex set in $\sigma_{0}$ containing $x$, with $\vec{V}_{0} \subset U \cap \sigma_{0}$. Let $\left\{\sigma_{i}\right\}_{1}^{\infty}$ be the collection of minimal simplices of $\operatorname{sd} K$ having $\sigma_{0}$ as a proper face, i.e., $\operatorname{dim} \sigma_{i}=\operatorname{dim} \sigma_{0}+1$ for all $i$. For $i \geqslant 1$, inductively choose $V_{i}$ to be a relatively open convex set in $\left|\sigma_{i}\right|$ such that:

(i) $V_{0} \subset \nabla_{i} \subset U \cap \sigma_{i} \cup V_{0}$,

(ii) $\nabla_{i} \supset \overline{\overline{\operatorname{conv}}}\left(\bigcup_{j<i} \nabla_{j}\right) \cap \sigma_{i}$,

(iii) $p_{i}\left(\nabla_{i}\right) \subset V_{0}$, where $p_{i}: \sigma_{i} \cup \sigma_{0} \rightarrow \sigma_{0}$ is the projection onto $\sigma_{0}$,

(iv) $\overline{\operatorname{conv}}\left(\bigcup_{j \leqslant i} V_{j}\right) \subset U$,

(v) $\overline{\operatorname{conv}}\left(\bigcup_{j \leqslant i} V_{j}\right) \cap \sigma_{k}=\overline{\operatorname{conv}}\left(\bigcup_{j<i} V_{j}\right) \cap \sigma_{k}$, for all $k<i$,

(vi) $\overline{\operatorname{con} \nabla}\left(\bigcup_{j \leqslant i} \nabla_{j}\right) \frown \sigma_{m} \subset p_{m}^{-1}\left(\nabla_{0}\right)$, for all $m>i$.

Then $V=\operatorname{con} v \bigcup_{1}^{\infty} \nabla_{i}$ is an open convex subset of $U \cap$ st $\sigma_{0}$ containing $x$ (note that $V \cap|\sigma|=\operatorname{conv} \bigcup\left\{\nabla_{i}:\left|\sigma_{i}\right| \subset|\sigma|\right\}$ ), and we show that $r(V) \subset V$. For $y \in V$, we have $y \epsilon \sigma \epsilon \operatorname{sd} K$, and $|\sigma|=\operatorname{conv}\left(\left|\sigma_{L}\right| \cup\left|\sigma^{*}\right|\right)$, where $\left|\sigma_{0}\right| \subset\left|\sigma_{L}\right| \subset|L|$ and $\left|\sigma^{*}\right| \cap|L|=\left|\sigma_{0}\right|$. Thus $y=a y_{L}+(1-a) y^{*}$, with $y_{L}$ $\epsilon$ conv $\bigcup\left\{\nabla_{i}:\left|\sigma_{i}\right| \subset\left|\sigma_{L}\right|\right\}$ and $y^{*} \epsilon \operatorname{con} \nabla \bigcup\left\{\nabla_{i}:\left|\sigma_{i}\right| \subset\left|\sigma^{*}\right|\right\}$. It is easily verified that $r|| \sigma|:| \sigma|\rightarrow| \sigma_{L} \mid$ maps intervals onto intervals. Thus $r(y)$ $=b r\left(y_{L}\right)+(1-b) r\left(y^{*}\right)=b y_{L}+(1-b) y_{0}$, for some $b \in I, y_{0} \in \nabla_{0}$, and $r(y)$. $\epsilon \operatorname{conv} \bigcup\left\{V_{i}:\left|\sigma_{i}\right| \subset\left|\sigma_{L}\right|\right\} \subset \nabla$.

CoROLLARY 4.7. Every countable CW polytope is strictly LEC.

THEOREM 4.8. Every $\mathrm{CW}$ polytope in which every 1-simplex is a face of only countably many simplices is strictly LEC.

Proof. Clearly, we may assume each vertex of such a polytope $K$ is a face of a 1-simplex. Introduce an equivalence relation $\sim$ on the set $\left\{e_{a}\right\}$

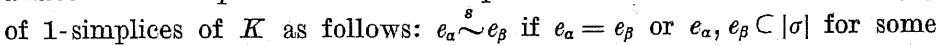
simplex $\sigma \in K$, and $e_{\alpha} \sim e_{\beta}$ if there exists a chain $\alpha=a_{1}, a_{2}, \ldots, \alpha_{n}=\beta$

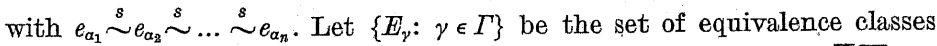
of 1 -simplices; each $E_{\gamma}$ is countable. For each $\gamma$, define $K_{\gamma} \equiv \overline{\operatorname{st}}\left(E_{\gamma}\right)$ $\left.=\bigcup \overline{\text { st } e}: e \in E_{\gamma}\right\}$. The 1-simplices of $K_{\gamma}$ are precisely the members of $E_{\gamma}$, and $\left|K_{\gamma}\right|$ is a countable connected subpolytope of $|K|$. Let $\lambda_{\gamma}: U_{\gamma} \times I \rightarrow\left|K_{\gamma}\right|$ be the strict local equiconnecting function obtained by (4.7). Define $U=\bigcup U_{\gamma} \cup \bigcup\left\{\operatorname{st}\left(v, K_{\gamma_{1}}\right) \times \operatorname{st}\left(v, K_{\gamma_{2}}\right): \gamma_{1} \neq \gamma_{2}, v \in K_{\gamma_{1}} \cap K_{\gamma_{2}}\right\}$. Then $U$ is a neighborhood of the diagonal in $|K| \times|K|$, and since the restriction 
of $\lambda_{\gamma}$ to each simplex of $K_{\gamma}$ is linear, we may define $\lambda: U \times I \rightarrow|K|$ as follows: $\lambda / U_{\gamma} \times I=\lambda_{y}$, and for $x_{i} \in \operatorname{st}\left(v, K_{\gamma_{i}}\right), i=1,2$, with $x_{i}(v)>0$ the $v$-barycentric coordinates, $\lambda\left(x_{1}, x_{2}, 0\right)=x_{1}, \lambda\left(x_{1}, x_{2},\left(1-x_{1}(v)\right) /\left(2-x_{1}(v)-\right.\right.$ $\left.-x_{2}(v)\right) \mid=v$, and $\lambda\left(x_{1}, x_{2}, 1\right)=x_{2}$, with $\lambda\left(x_{1}, x_{2}, t\right)$ extended piecewiselinearly on $I$. Then $\lambda$ is a strict local equiconnecting function for $|K|$.

Let $J_{i}=\left[-2^{-i}, 2^{-i}\right]$ and $J^{\infty}=\prod_{1}^{\infty} J_{i}$, with metric $d(x, y)=\max \left|x_{i}-y_{i}\right|$. Then $J^{\infty}$ is a copy of the Hilbert cube $I^{\infty}$. Let $R$ be the equivalence relation in $J^{\infty}$ defined by $x R y$ iff $x=y$ or $x=-y$, and consider the metrizable quotient space $J^{\infty} / R$. The image of $x \epsilon J^{\infty}$ under the natural projection $J^{\infty} \rightarrow J^{\infty} / R$ will be denoted by [x]. Schori [9] showed that $J^{\infty} \mid R \times I^{\infty}$ is homeomorphic to $I^{\infty}(2)$, the hyperspace of all non-void subsets of $I^{\infty}$ containing at most 2 points, and asked whether $J^{\infty} / R$, or $J^{\infty} / R \times I^{\infty}$, is homeomorphic to $I^{\infty}$. Wong subsequently noted that $J^{\infty} \mid R \backslash[0]$ is not simply connected, hence $J^{\infty} / R$ cannot be homeomorphic to $I^{\infty}$. Whether $J^{\infty} / R \times I^{\infty}$ is homeomorphic to $I^{\infty}$ remains an open question. Here, we show that $J^{\infty} / R$ is at least an $\operatorname{AR}(\mathfrak{M})$.

THEOREM 4.9. $J^{\infty} / R$ is strictly EC.

Proof. Define $\lambda: J^{\infty} / R \times J^{\infty} / R \times J \rightarrow J^{\infty} / R$ as follows: if $[y]=[0]$,

$$
\lambda([x],[y], t)=\left[\begin{array}{ll}
{[(1-2 t) x],} & 0 \leqslant t \leqslant \frac{1}{2} \\
{[0],} & \frac{1}{2} \leqslant t \leqslant 1
\end{array}\right.
$$

Otherwise, with $m=\log _{2}(d(x,-y) / d(x, y))$, and $\operatorname{sgn} m=1$ if $m \geqslant 0,-1$ if $m<0$,

$\lambda([x],[y], t)=\left\{\begin{array}{r}{[(\operatorname{sgn} m) t y+(1-t) x],|m| \geqslant 1,} \\ {[2 t m y /(1+|m|)+(1-2 t /(1+|m|)) x],} \\ 0 \leqslant t \leqslant(1+|m|) / 2,|m| \leqslant 1, \\ {[(\operatorname{sgn} m)(2 t-(1+|m|)) y /(1-|m|)+(2-2 t) m y /(1-|m|)],} \\ (1+|m|) / 2 \leqslant t \leqslant 1,|m| \leqslant 1 .\end{array}\right.$

It is easily verified that $\lambda$ is a well-defined continuous equiconnecting function, and that $J^{\infty} / R$ has an open base of $\lambda$-convex subsets.

Added in proof. Schori and Barit have recently shown that $J^{\infty} / R \times I^{\infty}$ is homeomorphic to $I^{\infty}$.

\section{References}

[1] K. Borsuk, Theory of retracts, Warszawa 1967.

[2] J. Dugundji, Note on CW polytopes, Port. Math. 11 (1952), pp. 7-106.

[3] - Locally equiconnected spaces and absolute neighborhood retracts, Fund. Math. 57 (1965), pp. 187-193.

[4] R. H. Fox, On fiber spaces, $I I$, Bull. Amer. Math. Soc. 49 (1943), pp. 733-735.

[5] 0 . Hanner, Retraction and extension of mappings of metric and non-metric spaces, Ark. Mat. 2 (1952), pp. 315-360.

[6] C. J. Himmelberg, Some theorems on equiconnected and locally equiconnected spaces, Trans. Amer. Math. Soc. 115 (1965), pp. 43-53.

[7] E. Michael, Local properties of topological spaces, Duke Math. J. 21 (1954), pp. 163-171.

[8] - Convex structures and continuous selections, Can. J. Math. 11 (1959), pp. 556-575.

[9] R. M. Schori, Hyperspaces and symmetric products of topological spaces, Fund. Math. 63 (1968), pp. 77-88.

\section{LOUISIANA STATE UNIVERSITY}

Baton Rouge, Louisiana

Reçu par la Rédaction le 3. 3. $19 \%$ 